\title{
Simulação no Contexto de Problemas Mal Estruturados
}

\author{
Antônio Sérgio de Souza \\ Marcos Augusto da Silveira \\ Mônica Santos de Pontes \\ Olympio de Andrade Jr.
}

Ladesp - Laboratório de Desenvolvimento de Sistemas de Produção

Departamento de Engenharia de Produção

Escola de Engenharia da Universidade Federal de Minas Gerais

Rua Espírito Santo, $35-7^{\circ}$ andar - Centro

30160-030 - Belo Horizonte - MG

\section{Wagner Barbosa}

Belgo Mineira Sistemas - BMS

Palavras-chave: Simulação; Modelamento; VIM.

Key Words: Simulation; Modelling; VIM - Visual interactive Modelling

\section{RESUMO}

Simulação é atualmente reconhecida como uma poderosa ferramenta no projeto, análise e operação de sistemas produtivos. Apesar dos avanços recentes em linguagens de simulação, interfaces gráficas e geradores de aplicações, simulą̧ão ainda é uma técnica cara e pouco eficiente. Discute-se no texto algumas causas deste fato no contexto de problemas mal estruturados, como é o caso de planejamento e controle da produção. Discute-se a necessidade de realização da modelagem conceitual, não apenas como um passo estanque no processo de simulação, mas de forma progressiva e dinâmica, durante todo o ciclo de vida do projeto, servindo como uma interface cntre os contextos real e de simulação.

\footnotetext{
ABSTRACT

Simulation is now recognized as powerfull tool in project, analyses and operation of produtive systems. Although the recent advances in simulation languages, graphical interfaces and in generating applications, simulation is still an expensive and not so efficient technique. In this text we dicuss some reasons for this in the context of not well strutured problems, asit is the case of production planning and control. It is also dicussed the necessity of developing the conceptual modeling of the real system, not only as a separated step in the simulation process, but in a progressive and dynamic way, during the project's life cycle as an interface between the real system and simulation.
} 


\section{Introdução}

No mercado econômico mundial, os setores operacionais estão sendo reconhecidos como os verdadeiros condutores das vantagens competitivas das empresas no cumprimento das datas de entrega, redução dos prazos de atendimento e maior flexibilidade. Tudo isto, sem perda dos padrões de qualidade e custo.

Sem dúvida, a dinâmica dos sistemas de produção, face a estas exigências, é extremamente complexa e de difícil tratamento analítico. Ademais, cada sistema de manufatura apresenta uma arquitetura única, decorrente do desenvolvimento de habilidades especificas, e da forma como são integradas (WICHMANN, 1990). Simulação aparece então como uma poderosa abordagem, que vem sendo utilizada com sucesso, nesta classe de problemas pouco estruturados.

\section{O Processo de Simulação}

Embora intimamente ligada aos computadores, a simulação não é, em sua essência, uma atividade computacional. Simulação busca obter um modelo capaz de reproduzir as respostas de sistemas reais, ou que podem se tornar reais, a eventos que ocorrem no tempo. É um processo cuja entrada é um problema e cuja saída é um modelo. Com a simulação, é possivel estimar parâmetros futuros consistentes com o estado atual do modelo. Apesar de que a sua utilização comercial tenha se beneficiado nos últimos anos de desenvolvimentos computacionais, ainda existe uma necessidade muito grande de habilidades es- pecificas, o que de alguma forma separa os contextos real (clientes) e de simulação (especialistas).

Há uma necessidade de se entender como obter um processo de simulação eficiente; que resulte em menor custo e maiores confiabilidade e flexibilidade do modelo. Discute-se, a seguir, as diversas fases do ciclo de vida de projetos de simulação caracterizando, sem pretender esgotar, aspectos que restringem a utilização mais ampla da simulação, principalmente no contexto de planejamento e controle da produção.

\section{Análise do Problema}

Em geral, os responsáveis pela supervisão de uma unidade produtiva observam resultados indesejáveis: o problema. Ferramentas corno o diagrama causa-efeito podem ser utilizadas na busca das causas mais importantes dos resultados indesejados. Conhecidas as causas, busca-se uma solução adequada, e questiona-se sobre o emprego da simulação. Considere os seguintes resultados indesejáveis (problemas) em uma linha de produção:

\section{1. tempo ocioso em equipamentos;}

2. baixo desempenho nos prazos de entrega.

Analisando o primeiro problema, verificou-se que a sua causa principal era a falta de dispositivos de transporte. A partir da elaboração de um modelo, trabalhou-se dados coletados e estimados, e o resultado obtido através da simulação foi facilmente entendido e implantado. As causas encontradas no segundo problema foram várias, envolvendo diversas áreas da empresa: vendas, finanças, planeja- 
mento da produção, controle da produção e supervisão do chão-de-fábrica; cada área com atividades e objetivos especificos de fixar prazos de entrega suficientemente competitivos, definir níveis máximos de estoques de material em processo, elaborar o programa mestre de produção que atenda às exigências de disponibilidade para entrega necessária para vendas, programar frequência de recebimentos, definir políticas de despacho da produção, visando niveis de utilização de equipamentos e cumprimento dos prazos de entrega.

A formulação do segundo problema é pobre, resultado de sugestões e expectativas conflitantes. O resultado é questionado e de difícil implantação. Oprimeiro problema, bem estruturado e resolvido com sucesso via simulação, é na realidade o segundo problema visto por uma ótica "fechada". Neste contexto, mais amplo, a resolução do primeiro problema só é relevante se os equipamentos ociosos forem gargalo de produção. Caso contrário, sua resolução pode levar a aumentos de estoque de material em processo e do tempo de fluxo, ou seja, ao agravamento do segundo problema.

"Fechar" os problemas e iniciar apressadamente um projeto de simulação, pode levar a custos desnecessários e um alto risco de insucesso. Por outro lado, o excesso de "abertura" pode minar a confiança da administração, sempre impaciente por resultados.

Uma possivel abordagem a este dilema, é a realização, desde o início, da modelagem conceitual, normalmente um passo estanque (nem sempre realizado) nos projetos de simulação. Existem representações (ICAM, CIM-OSA, GRAI, entre outras), que podem ser facilmente entendidas por pessoas de áreas diferentes, que são independentes de softwares e plataformas, e que são adequadas a um processo de refinamentos sucessivos. SCHEER (1992), WANG (1993), discutem algumas representações.

A modelagem conceitual é geralmente confundida com a montagem do modelo na linguagem de simulação. A modelagem conceitual é um processo de captura de uma descrição de uma estrutura do domínio de aplicação, realizada em uma representação estruturada, e que sirva de referência no contexto da simulação.

Para evitar redundância de dados e estruturar a complexidade dos problemas, a modelagem conceitual é realizada em um conjunto de visões sobre o domínio de aplicação. Um conjunto mínimo pode ser definido com uma visão estática (de dados) e outra dinâmica (das funções e atividades). A representação proposta pela USAF (United States Air Force) no projeto ICAM (Integrated Computer-Aided Manufacturing), propõe uma série de visões, com as respectivas metodologias $\mathrm{IDEF}_{x}$ (ICAM Definitions), que possuem características de decomposição, ou seja, atividades de um nivel mais alto podem ser "explodidas" em atividades de niveis menores.

No passo inicial, de análise do problema, a visão funcional é suficiente e pode ser obtida pela metodologia $\mathrm{DEF}_{0}$ (ICAM Definitions Functions). Esta metodologia propõe representações gráficas em sucessivos refinamentos, é disponível em software, e é bastante apropriada para a descrição das atividades e funções do planejamento e controle da produção.

A análise do problema é crucial para o sucesso da simulação, que é uma técnica cara e que consome muito tempo. Estar seguro de que nenhuma outra técnica pode ser utilizada, e que o problema a ser resolvido contribui 
para uma maior eficácia do sistema produtivo em relação aos objetivos estratégicos dos sistemas produtivos é, portanto, fundamental. $\mathrm{E}$ isto, principalmente, para se obter o apoio interno, sem o qual o projeto está fadado ao insucesso.

A modelagem conceitual é útil para se obter o apoio da alta gerência, geralmente sensível apenas aos parâmetros como ROI (Return on Investment), por permitir uma justificativa estratégica e consistente para o projeto. É fundamental, no entanto, boas estimativas de custos de desenvolvimento do projeto e receitas a serem obtidas com as reduções de estoque, atraso de pedido e no aumento de produtividade, entre outros. $\mathrm{O}$ apoio interno deve vir de uma reunião formal, onde o projeto é discutido e, se nada der errado, aprovado formalmente com o comprometimento da alta gerência.

\section{Definição do Software, Desenvolvimento de Piloto e Validação.}

É pratica comum que, uma vez aprovado o projeto, o software seja logo adquirido para que a equipe possa dominá-lo o mais rapidamente possivel. Esta prática pode levar a adquirir um produto que não permita a realização do modelo concebido, na complexidade necessária. $O$ compromisso entre flexibilidade ou rapidez no desenvolvimento do modelo deve ser considerado. Geralmente, linguagens de simulação oferecem maior flexibilidade, ao passo que os "simuladores de fábrica" permitem um desenvolvimento mais rápido.
Ao contrário da prática, a definição do software deve ser orientada pelas necessidades definidas após refinamentos do modelo conceitual. As atividades e funções definidas na visão funcional, durante a análise do problema, são desdobradas em atividades detalhadas e bem definidas como, por exemplo, algoritmos de liberação de ordens e tratamento de paradas de máquinas.

Nesta fase, deve-se também acrescentar ao modelo conceitual a visão estática de dados, relativa aos objetos e informações existentes. A metodologia $\mathrm{DEF}_{1}$, (ICAM Definitions Informations) que descreve os dados em uma forma bastante próxima de entidade-relacionamento, é bastante adequada e eficiente na geração da estrutura de dados. No caso da simulação aplicada ao planejamento e controle da produção, cujo resultado é utilizado freqüentemente, e não apenas uma única vez, a visão de dados serve também para orientar na compra do software a ser adquirido, com relação às necessidades de relacionamento com outros sistemas já existentes e, principalmente, para importação e exportação de dados.

Embora a visão funcional mostre implicitamente a sequiência de atividades, eventos gerados e necessidades de sincronismos, pode ser necessário uma terceira visão, explicitamente dinâmica, possivelmente mais próxima das representações de linguagens de simulação, que descreva o comportamento temporal entre as funções e os objetos do sistema. Regras de produção, por exemplo, são representações bastante próximas de linguagens de simulação orientadas a atividades. Pode-se especificar softwares que permitam a incorporação posterior da visão dinâmica como bases de conhecimento. A Figura 1 ilustra as visões do modelo conceitual descrito. 


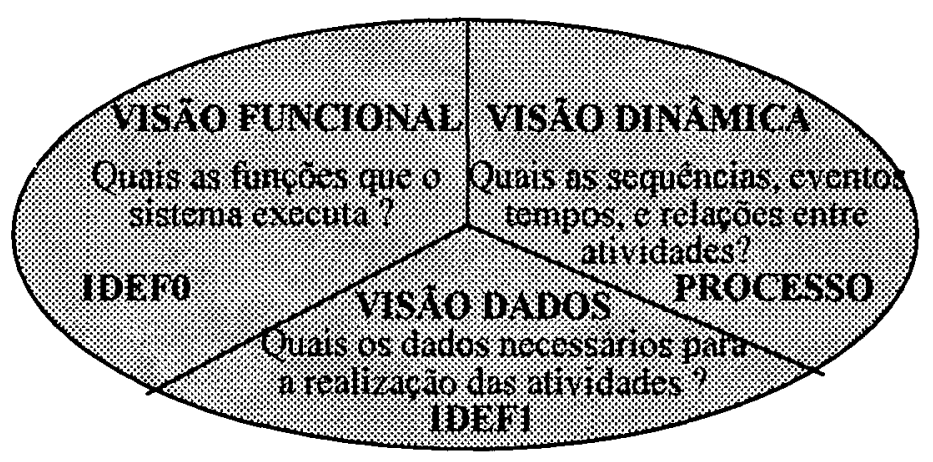

Figura 1 Visões de um modelo conceitual

na escolha do momento adequado para adotar sugestões e buscar discuti-las com base no modelo conceitual, que permite uma visão mais clara do impacto de cada modificação, em todas as áreas envolvidas. Problemas na implantação podem ocorrer pela falta de dedicação do geren-

A realização de um projeto piloto enriquece o projeto, permitindo avaliar a equipe, os recursos disponiveis e a real complexidade do problema. A preocupação deve ser para a simplicidade do piloto, de tal forma a não alongar o projeto. A validação é uma etapa para se testar a lógica e os dados utilizados. Geralmente, contrapõe-se os resultados aos dados históricos.

\section{Projeto de}

\section{Experimento,}

\section{Desenvolvimento}

\section{Final e Implantação}

O desenvolvimento do produto final e do projeto de experimento deve considerar a necessidade de mudanças e modificações posteriores (manutenção). Este aspecto é particularmente importante no caso da simulação aplicada ao planejamento e controle da produção, cujo resultado será utilizado freqüentemente e não apenas uma única vez. Em geral, os "clientes" visualizam, logo após os primeiros testes, melhorias que, embora válidas se forem acatadas, tornam o processo de simulação sem fim. Deve-se tomar cuidado te de projeto.

\section{Conclusões}

Procurou-se mostrar as dificuldades no processo de simulação, e sugeriu-se algumas abordagens. $O$ aspecto mais importante é a necessidade de mecanismos entre os contextos real e da simulação. A extensão da fase de modelagem conceitual para todo o ciclo de vida do projeto pode ser uma solução, tornando possivel que as diversas áreas (clientes), cada uma com seus objetivos específicos, e os especialistas cooperem, durante todo o ciclo de vida do projeto, para o desenvolvimento progressivo de um modelo aberto. Os resultados são maior consistência entre as áreas e aderência aos objetivos estratégicos da empresa. Ademais, o modelo conceitual é em si um produto que pode ser reutilizado em outros projetos, permitindo um conjunto consistente de aplicações. Em resumo, simulação pode ser mais que a obtenção de resultados númericos estatisticamente válidos. Sendo um processo de obtenção de um modelo, pode promover uma mudança de uma ótica funcional para uma mais global, desenvolvendo novas habilidades internas na empresa, o que no finalé a real fonte de vantagem competitiva. 


\section{Anexo}

\section{Técnicas de Modelagem IDEF}

Uma das principais características desta técnica é sua capacidade de decomposição, isto é, atividades de um nivel mais alto podem ser explodidas em atividades de um nivel menor, criando assim novos diagramas. A cada diagrama é atribuído umúmero, que define onde este diagrama se encaixa na hierarquia do modelo. Um modelo IDEF compreende um conjunto de diagramas de atividades relacionadas, ordenados pelo seu número correspondente, que representam o tema em estudo. A abordagem IDEF trabalha, entre outras coisas, com as caracteristicas funcionais $\left(\mathrm{IDEF}_{0}\right) \mathrm{e}$ informações $\left(\mathrm{IDEF}_{1}\right)$ do modelo (SARKIS, 1994).

$\mathrm{DEF}_{0}$ é uma técnica de modelagem funcional, utilizada para proporcionar ao usuário um meio de entendimento do processo de produção, facilitando a comunicação entre a parte de produção (chão-de-fábrica) e desenvolvedores do sistema. Ela é utilizada não somente no trabalho técnico de definição e projeto de sistemas, mas também no gerenciamento e integração do projeto. O modelo consiste basicamente de diagramas hierarquicamente relacionados. Cada diagrama possui uma linha diagonal principal de blocos (normalmente de 3 a 6 blocos), conectados através de setas. Este processo é mostrado na Figura A1. Os blocos representam as atividades a serem executadas, funções ou processos. As setas representam o relacionamento entre as atividades, de acordo com a informação ou material utilizado, produzido ou requerido pelas atividades. As setas que entram do lado esquerdo do bloco representam a entrada de eventos, chegada de materiais ou informações, e é o que habilita a atividade. As setas que saem do lado direito do bloco representam a saída de eventos, ou seja, saída de informações ou materiais, que é o que é gerado pela execução da atividade. As setas que entram no topo do bloco representam as condições/restrições da atividade. $\mathrm{E}$, finalmente, as setas que entram na base do bloco, representam os recursos (por exemplo homens, máquinas, técnicas e softwares), meios necessários para a realização da atividade.

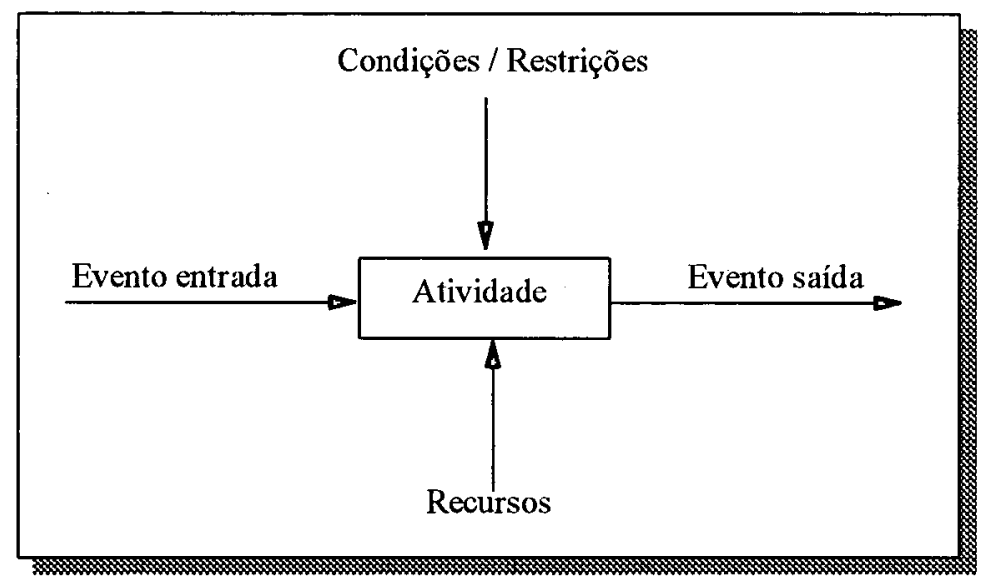

Figura A1- Metodologia IDEF。 
DEF, é uma técnica de modelamento basedo na informação, e segue os conceitos de modelagem IDEF. Os modelos de informação são usados para identificar as entidades relevantes e os relacionamentos existentes para criar as várias tabelas do banco de dados que as contém.

$\mathrm{Na}$ aplicação a seguir, tem-se um exemplo de aplicação da técnica de modelagem IDEF para uma empresa metal-mecânica:

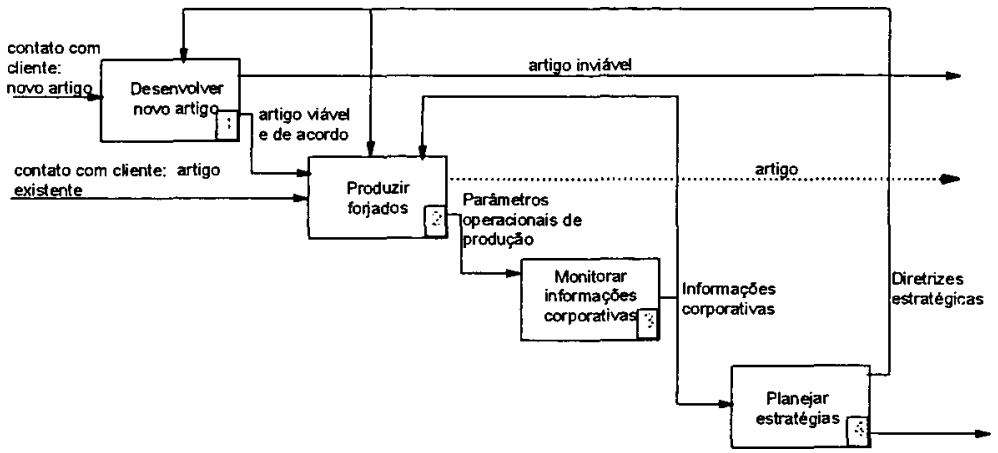

\begin{tabular}{l|l} 
Diagrama: A4 & Processo: Do contato com cliente à entrega do produto
\end{tabular}

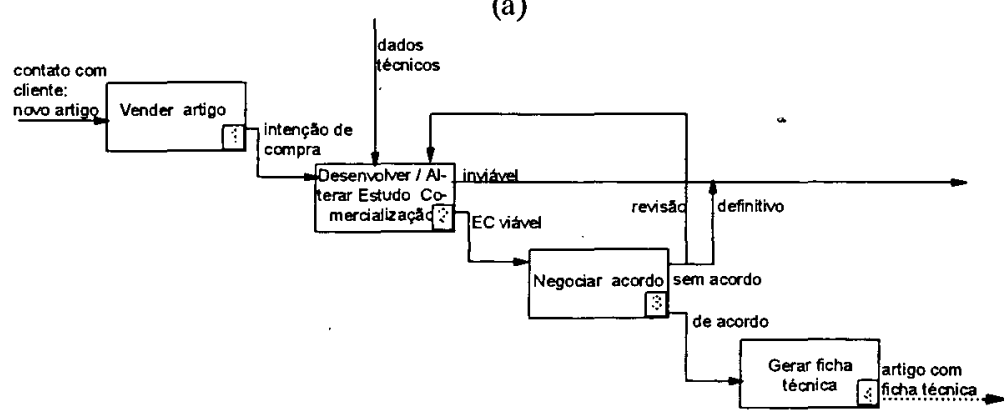

Diagrama: A4

Processo: Do contato com cliente à entrega do produto

(b)

Figura A2 - Modelo IDEF,

Observe que a Figura A2 (b) é um detalhamento do bloco 1 do diagrama da Figura A2(a). 


\section{Referências}

\section{bibliograficas}

SARKIS, J; LIN,L. An IDEF0 functional planning model for the strategic implementation of CIM systems. Int. Journal Computer Integrated Manufacturing, 1994, vol.7, n², 100-115.

SCHEER, A. Architeture of Integrated Information Systems. Spring-Verlag, 1992.
WICHMANN, K. E. Simulation-based production schedule. Production Planning and Control, vol. 1, n³ 3, 179-189, 1990.

WANG, W; POPPEWELL,K; BELL,R.An integrated mutli-view system description approach to approximate factory modelling. Int. Journal Computer Integrated Manufacturing, 1993, vol.6, $\mathrm{n}^{\circ} 3$, 165-174. 